\title{
Estudo comparativo entre oclusão e não-oclusão no tratamento de abrasão corneana após retirada de corpo estranho da córnea
}

\author{
Comparative study between eyepad and no eyepad in the treatment of corneal abrasion \\ secondary to removal of corneal foreign body
}

\author{
Rafael Mourão Agostini' ${ }^{1}$ \\ Guilherme Mourão Soares da Rocha² \\ Jane Claudia Miranda² \\ Daniela Vieira de Ag'uiar ${ }^{1}$ \\ João Agostini Netto ${ }^{3}$
}

Trabalho realizado na Clínica de Olhos da Santa Casa de Belo Horizonte.

${ }^{1}$ Médicos Residentes do terceiro ano de Oftalmologia da Clínica de Olhos da Santa Casa de Belo Horizonte.

${ }^{2}$ Médicos Residentes do segundo ano de Oftalmologia da Clínica de Olhos da Santa Casa de Belo Horizonte.

${ }^{3}$ Chefe da Clínica de Olhos da Santa Casa de Belo Horizonte. Professor Titular da Disciplina de Oftalmologia da Faculdade de Ciências Médicas de Minas Gerais.

Endereço para correspondências: Rafael Mourão Agostini, Rua Ceará $\mathrm{n}^{\circ} 518$ - $2^{\circ}$ andar - Belo Horizonte (MG) CEP 30150-310

E-mail: rafaelagostini@uol.com.br

Recebido para publicação em 09.01.2003

Versão revisada recebida em 17.07.2003

Aprovação em 07.08.2003

Nota Editorial: Pela análise deste trabalho e por sua anuência na divulgação desta nota, agradecemos aos Drs. Paiva Gonçalves Filho e Paulo Ricardo de Oliveira.

\section{RESUMO}

Objetivo: Comparar a eficácia entre duas modalidades de tratamento das abrasões corneanas após a retirada de corpo estranho da córnea: curativo oclusivo e sem curativo. Métodos: Cinqüenta e quatro pacientes com abrasão corneana após retirada de corpo estranho foram randomizados, de forma alternada, em dois grupos: um grupo com curativo oclusivo e o outro sem curativo. Os pacientes foram avaliados diariamente até a cura, em relação aos seguintes parâmetros: área da abrasão corneana, intensidade de dor, presença de fotofobia, lacrimejamento, sensação de corpo estranho e visão turva. Resultados: Não houve diferença estatisticamente significativa entre os dois grupos quanto a: área da abrasão corneana, tempo para se obter a cura, dor, fotofobia, lacrimejamento, sensação de corpo estranho e visão turva. Não ocorreu nenhuma complicação ocular ou sistêmica durante o tratamento em ambos os grupos. Conclusão: Abrasão corneana após retirada de corpo estranho, menor que $9 \mathrm{~mm}^{2}$, pode ser tratada apenas com antibiótico tópico de largo espectro e colírio cicloplégico, sem a necessidade do curativo oclusivo, tornando o tratamento mais simples e menos dispendioso.

Descritores: Traumatismos oculares/terapia; Córnea/lesões; Corpos estranhos no olho/ terapia; Cicatrização de feridas

INTRODUÇÃ̃O

Abrasão corneana após retirada de corpo estranho é uma das mais freqüentes ocorrências nos serviços oftalmológicos ${ }^{(1)}$.

A córnea é ricamente inervada, e estando estas terminações nervosas livres no epitélio ${ }^{(2)}$, qualquer erosão corneana, mesmo as superficiais, são muito sintomáticas (dor, sensação de corpo estranho e embaçamento visual). Pelo fato do defeito reepitelizar-se, geralmente em 2 a 3 dias, pouco tem sido pesquisado sobre as formas de tratamento ${ }^{(3)}$.

A conduta comumente empregada é: retirada do corpo estranho, com seu halo de impregnação, se de natureza metálica, uso de antibiótico tópico, colírio cicloplégico e curativo oclusivo.

Recentes estudos têm questionado a terapia oclusiva. Kayser e cols. concluíram que pacientes curavam-se significativamente mais rápido e tinham menos dor e sintomas quando não recebiam curativo oclusivo ${ }^{(4-5)}$.

Este trabalho, prospectivo, tem como objetivo analisar e comparar duas formas de terapia: com curativo oclusivo e sem curativo oclusivo. 


\section{MÉTODOS}

O estudo foi aprovado pela Comissão de Ética e Pesquisa da SCBH. Foram incluídos no trabalho pacientes atendidos no Setor de Urgências da Clínica de Olhos da Santa Casa de Belo Horizonte (SCBH), entre primeiro de julho e 31 de outubro de 2001, com diagnóstico de corpo estranho corneano não orgânico, e com menos de 48 horas de evolução. Os pacientes, uma vez informados sobre o estudo, eram avaliados quanto aos seguintes critérios de inclusão: idade maior ou igual a 18 anos; sem sinais de outro trauma ocular; presença de corpo estranho corneano sem sinais de infiltrado peri lesional; ausência de história de doença ocular com comprometimento corneano; e não ser usuário de lente de contato.

Todos os pacientes foram submetidos a uma completa anamnese e exame oftalmológico. Após a administração de colírio anestésico (Allergan ${ }^{\circledR}$ - cloridrato de tetracaína $100 \mathrm{mg}$ e cloridrato de fenilefrina $1 \mathrm{mg}$ ), fazia-se a remoção do corpo estranho e, se necessário, do seu halo de impregnação metálica com um removedor de halo corneano (Algerbrush ${ }^{\circledast}$ II com ponteira de $0,5 \mathrm{~mm}$ ). Em seguida, instilava-se uma gota de fluoresceína sódica $1 \%$ (Allergan ${ }^{\circledR}$ ) no fórnice conjuntival inferior do olho acometido e fazia-se a medida do maior diâmetro vertical e horizontal da abrasão corneana com auxílio da lâmpada de fenda - DF Vasconcelos.

Os pacientes foram aleatoriamente separados em dois grupos: o com oclusão (C.O.), no qual os pacientes recebiam curativo oclusivo, e o grupo sem oclusão (S.O.), que não recebia curativo. Nos dois grupos, após a retirada do corpo estranho e do halo de impregnação, a seguinte rotina foi seguida: medição da área de erosão corada com fluoresceína, limpeza ocular com soro fisiológico, administração de uma gota de cloridrato de ciclopentolato $1 \%$ (Allergan ${ }^{\circledR}$ ) e aplicação de pomada oftálmica Epitezan ${ }^{\circledR}$ (Acetato de retinol 10.000UI, cloranfenicol $5 \mathrm{mg}$, metionina $5 \mathrm{mg}$, aminoácidos $25 \mathrm{mg}$ ). Após esse procedimento, os pacientes eram submetidos, de forma alternada, à terapia oclusiva. $\mathrm{O}$ curativo era feito pela equipe de enfermagem da Clínica de Olhos - SCBH, usando compressas de gaze estéril (Cirumex ${ }^{\circledR}$ ) e esparadrapo hipoalergênico, de modo que impedisse o piscar sob o curativo. Os pacientes do grupo C.O. eram instruídos a deixar o curativo por 24 horas, até o retorno ao hospital. O grupo S.O. foi orientado a usar a pomada oftálmica Epitezan ${ }^{\circledast}$ três vezes ao dia, durante cinco dias ou até o fechamento do defeito epitelial. Os pacientes do grupo C.O. que não preenchiam os critérios de cura no primeiro retorno, recebiam novo curativo com pomada Epitezan ${ }^{\circledR}$, a cada 24 horas, até a cura. Os dois grupos foram liberados para utilizar analgésicos e antiinflamatórios orais.

Todos os pacientes retornavam diariamente ao hospital para responder sobre o uso correto da pomada no grupo S.O. e da permanência do curativo no grupo C.O. A intensidade da dor foi quantificada utilizando-se uma escala de dor $^{(4)}$, pela qual se atribuíam notas subjetivas entre 0 e 10 quando indagados, sendo que 0 (zero) correspondia à ausência de dor e 10 (dez) à dor muito intensa. Também foram indagados sobre a presença dos seguintes sintomas: fotofobia, lacrimejamento, sensação de corpo estranho e visão turva. Durante os dias de acompanhamento, que variou de um a três, a medida da área de erosão corneana foi realizada sempre pelos mesmos observadores. Os pacientes foram considerados curados de acordo com os seguintes critérios: escala de dor menor ou igual a dois e, ao exame com fluoresceína, apresentavam uma erosão leve, não confluente, superficial e ponteada do epitélio corneano.

\section{Analise Estatística}

Foram analisados pelo teste $\mathrm{t}$ de Student: idade dos pacientes; tempo de evolução antes do tratamento; área da erosão corneana; nota da escala de dor no dia da remoção do corpo estranho corneano e nos dias de tratamento; diferença da área da lesão do primeiro para o segundo dia; e dias necessários para a cura. Já a homogeneidade das amostras foi testada pelo teste de Fischer.

Os sintomas (fotofobia, lacrimejamento, sensação de corpo estranho e visão turva) foram analisados pelo teste do Quiquadrado não corrigido. Foram consideradas diferenças estatisticamente significativas quando o $p$ foi menor que $0,05(p<0,05)$.

\section{RESULTADOS}

Oitenta e dois pacientes foram incluídos no estudo. Vinte oito (34\%) foram excluídos ou, por não seguirem o tratamento corretamente (um paciente do grupo C.O. retirou o curativo antes das $24 \mathrm{~h}$ e três pacientes do grupo S.O. não usaram a pomada), ou por não retornarem nos dias subseqüentes (13 pacientes do grupo com oclusão e 11 do grupo sem oclusão). Dos 54 pacientes que se mantiveram no estudo, os homens representaram a maioria absoluta com 98\% dos casos (53homens e apenas uma mulher). A média de idade dos grupos foi de 34 anos.

Dos 54 pacientes, 27 foram do grupo C.O. e 27 do grupo S.O. A maior área da erosão corneana foi $9 \mathrm{~mm}^{2} \mathrm{e}$ a menor de $0,25 \mathrm{~mm}^{2}$. Nenhum paciente necessitou mais que três dias para a cura. Como ilustrado na tabela 1 não houve diferença significativa entre os dois grupos em relação à idade, tempo de evolução antes da apresentação, tamanho da erosão corneana e intensidade de dor antes do tratamento $(p>0,05)$. Também não houve diferença estatisticamente significativa entre os grupos após o tratamento quanto à nota dada na escala de dor, ao tamanho da erosão, à diferença da área da abrasão na apresentação e no dia seguinte e nem no tempo necessário para a cura. Com relação à sintomatologia, não houve diferença significativa entre os grupos quanto às queixas de fotofobia, lacrimejamento, sensação de corpo estranho e visão turva após instituição do tratamento (Tabela 2).

Durante o estudo não foi constatada nenhuma complicação sistêmica ou ocular em nenhum dos dois grupos. 


\begin{tabular}{|c|c|c|c|}
\hline Característica & Grupo controle & Grupo não ocluído & $P^{*}$ \\
\hline Número de pacientes & 27 & 27 & \\
\hline Idade (em anos) Média \pm desvio padrão & $35 \pm 13,22(18-77)$ & $33 \pm 10(18-56)$ & 0,633 \\
\hline Tempo de evolução (em horas) média \pm desvio padrão & $22 \pm 6,69$ & $26 \pm 11$ & 0,196 \\
\hline Área da erosão $\left(\mathrm{em} \mathrm{mm}^{2}\right)$ Média \pm desvio padrão & $1,22 \pm 0,89$ & $1,80 \pm 2,03$ & 0,180 \\
\hline Dor na apresentação Média \pm desvio padrão & $5,59 \pm 2,11$ & $6 \pm 1,86$ & 0,455 \\
\hline
\end{tabular}

\begin{tabular}{|c|c|c|c|}
\hline Achados & Grupo controle & Grupo não ocluído & $\boldsymbol{P}$ \\
\hline \multicolumn{4}{|l|}{ Escala de dor } \\
\hline Dia 2 (Média \pm desvio padrão) & $0,44 \pm 1,05$ & $0,96 \pm 2,24$ & 0,283 \\
\hline Dia 3 (Média \pm desvio padrão) & $0,11 \pm 0,57$ & $0,14 \pm 0,45$ & 0,794 \\
\hline \multicolumn{4}{|l|}{ Área de erosão $\left(\mathrm{mm}^{2}\right)$} \\
\hline Dia 2 (Média \pm desvio padrão) & $0,07 \pm 0,19$ & $0,10 \pm 0,20$ & 0,620 \\
\hline Dia 3 (Média \pm desvio padrão) & $0,00 \pm 0,00$ & $0,01 \pm 0,04$ & 0,167 \\
\hline Dias para cura & $1,22 \pm 0,42$ & $1,33 \pm 0,55$ & 0,411 \\
\hline Diferença na área de erosão entre dia 1 e $2\left(\mathrm{em} \mathrm{mm}^{2}\right)$ & $1,14 \pm 0,90$ & $1,70 \pm 1,89$ & 0,174 \\
\hline Fotofobia & $7(25 \%)$ & 13 (48\%) & $0,091^{*}$ \\
\hline Lacrimejamento & $10(37 \%)$ & $8(29 \%)$ & $0,563^{*}$ \\
\hline Sensação de corpo estranho & $7(25 \%)$ & $13(48 \%)$ & $0,091^{*}$ \\
\hline Visão borrada & $7(25 \%)$ & $8(29 \%)$ & $0,761^{*}$ \\
\hline
\end{tabular}

\section{DISCUSSÃO}

Nosso estudo é concordante com a literatura mundial no que diz respeito à predominância do sexo masculino e de jovens. Portanto, essas lesões afetam a população economicamente ativa, causando prejuízos, tanto pelo custo do tratamento, quanto pelo afastamento das atividades profissionais. Não entramos no mérito, fundamental, da prevenção desses acidentes, mas discutimos o melhor e menos dispendioso tratamento. Aquele que devolva mais precocemente esses indivíduos às suas tarefas produtivas.

Conforme sua extensão, os defeitos epiteliais corneanos são reparados por migração e proliferação das células próximas às margens da lesão ${ }^{(5-11)}$. Existem algumas opções de tratamento para a erosão corneana traumática, como a oclusão apenas do olho acometido, oclusão bilateral e o uso de lente de contato gelatinosa terapêutica. A oclusão bilateral, onde tanto o olho acometido quanto o olho adelfo são ocluídos, já demonstrou ser uma forma de tratamento que leva à rápida reepitelização corneana, provavelmente por reduzir o atrito da córnea com a conjuntiva tarsal ao inibir o ato de piscar ${ }^{(5-12)}$. Entretanto, requer o afastamento do indivíduo de suas atividades. O uso de lente de contato demonstrou ser eficaz no tratamento de abrasões corneanas, mas envolve alto custo e aumenta o risco de infecção, após a retirada de corpos estranhos possivelmente infectados ${ }^{(13)}$.

O estudo em questão demonstrou, como em outros estu$\operatorname{dos}^{(4-10)}$, que a oclusão palpebral no tratamento da erosão cor- neana após retirada de corpo estranho não otimiza a reepitelização e a cura. Abolido o ato de piscar, o atrito da conjuntiva tarsal sobre a córnea é minimizado pelo curativo oclusivo, entretanto não se observa a inibição completa da movimentação ocular sob as pálpebras. A oclusão palpebral parece não só provocar redução do "turn over" do filme lacrimal ${ }^{(8)}$, decréscimo na oferta de oxigênio ao epitélio corneano ${ }^{(6-7)}$, déficit da produção energética celular e acúmulo de produtos do metabolismo celular, mas também pode interferir com a flora bacteriana devido ao aumento da temperatura local ${ }^{(9)}$, conforme mostrado em alguns estudos.

Além disto, a oclusão monocular pode causar desconforto ao paciente, impede a visão binocular, com conseqüente redução do campo visual e da estereopsia, prolongando a reabilitação e oferecendo risco de novas lesões ${ }^{(10)}$.

A média da área de erosão do grupo C.O. foi menor que a do grupo S.O. $\left(1,22 \mathrm{~mm}^{2}\right.$ contra $1,80 \mathrm{~mm}^{2}$ com $\left.\mathrm{p}=0,180\right)$, apesar de não ser estatisticamente significativo. Isso poderia levar a um pior desempenho do grupo S.O. em relação à dor, tempo de cura e até em relação à sintomatologia, entretanto tal fato não foi observado. O que se notou foi uma maior incidência de fotofobia e sensação de corpo estranho ( $25 \%$ no grupo C.O. e $48 \%$ no grupo S.O. com $\mathrm{p}=0,091$ ) no grupo S.O., sem ser, entretanto, estatisticamente significativo, o que poderia ser atribuído à diferença no tamanho da erosão entre os grupos. Porém, tal diferença reverte-se a favor do grupo S.O., ao se constatar que todos os outros parâmetros avaliados, objetivos ou subjetivos, foram semelhantes entre os grupos. 
Deve-se observar que a maior área de abrasão corneana pós-retirada de corpo estranho encontrada neste estudo foi de $9 \mathrm{~mm}^{2}$, assim, a conclusão não deve, ainda, ser expandida para lesões de dimensões maiores, que necessitam de outros estudos. Esse fato foi observado num estudo ${ }^{(4)}$, no qual lesões maiores do que $10 \mathrm{~mm}^{2}$ necessitaram de tempo maior para a cura nos pacientes que não receberam terapia oclusiva, quando comparados ao grupo que recebia curativo oclusivo, apesar da diferença encontrada não ter sido estatisticamente significativa.

\section{CONCLUSÃO}

No presente estudo, o curativo oclusivo não acelerou a reepitelização corneana e nem proporcionou maior conforto aos pacientes. Sugere-se, então, que abrasão corneana, com diâmetro de até $9 \mathrm{~mm}$ após retirada de corpo estranho, possa ser tratada com antibiótico tópico de amplo espectro profilaticamente e colírio cicloplégico, abolindo-se a prática do curativo oclusivo. Erosões maiores devem ainda seguir a terapêutica tradicional, até que sejam avaliadas por novos estudos.

\section{AGRADECIMENTOS}

Dr. Carlos Rubens de Figueiredo e Dr. Emilio Rintaro Suzuki Junior - Médicos Assistentes da Clínica de Olhos da Santa Casa de Belo Horizonte - nossos agradecimentos pela colaboração na execução do trabalho.

\section{ABSTRACT}

Purpose: To compare the effectiveness of two forms of treatment of corneal abrasion secondary to removal of corneal foreign body: eyepad and no eyepad. Methods: Fifty-four patients with corneal abrasion secondary to removal of foreign body were randomized into two groups: the eyepad group and the no eyepad group. The patients were followed up daily, until complete healing, to evaluate: size of corneal abrasion, pain, photophobia, tearing, foreign body sensation, blurred vision. Results: There was no statistical difference between the two groups comparing: size of corneal abrasion, days until complete healing and amount of pain, photophobia, tearing, foreign body sensation or blurred vision. There were no systemic or ocular complications during the study. Conclusion: Corneal abrasion secondary to removal of foreign body, smaller than $9 \mathrm{~mm}^{2}$, can be treated with large spectrum topical antibiotic and cicloplegic drops alone without the need for eyepad, making treatment simpler and less expensive.

Keywords: Eye injuries; Cornea/injury; Eye foreign bodies/ therapy; Curatives occlusive; Occlusive dressings; Wound healing

\section{REFERÊNCIAS}

1. Chiapella AP, Rosenthal AR. One year in an eye casualty clinic. Br J Ophthalmol. 1985;69:865-70

2. Traumatic corneal abrasion. Lancet $1987 ; 2: 1250$

3. Kaiser PK, Pineda R. A study of topical nonsteroidal anti-inflammatory drops and no pressure patching in the treatment of corneal abrasions. Corneal Abrasion Patching Study Group. Ophthalmology 1997;104:1353-9

4. Kaiser PK. A comparison of pressure patching versus no patching for corneal abrasions due to trauma or foreign body removal. Corneal Abrasion Patching Study Group. Ophthalmology 1995;102:1936-42

5. Jackson H. Effect of eye-pads on healing of simple corneal abrasions. Br Med J $1960 ; 2: 713$

6. Weissman BA., Fatt I, Rasson J. Diffusion of oxygen in human corneas in vivo. Invest Ophthalmol Vis Sci 1981;20:123-5

7. Acheson JF, Joseph J, Spalton DJ. Use of soft contact lenses in an eye casualty department for the primary treatment of traumatic corneal abrasions. Br J Ophthalmol 1987;71:285-9

8. Milder B. The lacrimal aparatus. In: Moses RA, editor. Adler's physiology of the eye: clinical application. St. Louis: Mosby;1981. p.16-37.

9. Parrish CM., Chandler JW. Corneal trauma. In: Kaufman HE, Barron BA, Mc Donald, Waltman SR, editors. The cornea. New York, NY: Churchill Livingstone; 1988: p.599-646.

10. Hulbert MF. Efficacy of eyepad in corneal healing after corneal foreign body removal. Lancet 1991;337:643.

11. Kirkpatrick JN, Hoh HB, Cook SD. No eyepad for corneal abrasion. Eye 1993; 7:468-71.

12. Gregersen PL, Ottovay E, Kobayashi C, Hansen SE, Bohnstedt J. Treatment of abrasion corneal. Ugeskr Laeger 1991;153:2123-4.

13. DeBroff BM, Donahue SP, Caputo BJ. Azar MJ, Kowalski RP, Karenchak LM. Clinical characteristics of corneal foreign bodies and their associated culture results. CLAO J 1994; 20:128-30.

\title{
ABO ELETRÔNICO
}

\author{
Novo site
}

Acesso: http://www.abonet.com.br 